\title{
Keyword Extraction Methodologies Based on Rényi Entropy and Tsallis Relative Entropy
}

\author{
Aakanksha Singhal and D.K. Sharma \\ Department of Mathematics, Jaypee University of Engineering and Technology, \\ A.B. Road, Raghogarh, Dist. Guna (M.P)-473226, India. \\ Corresponding Author :dilipsharmajiet@gmail.com
}

\begin{abstract}
Textual and other forms of data is accumulating at a very fast pace through various social, academic and economic activities. Keyword extraction technique plays a significant role in analyzing, integrating, interpreting this amorphous data and exploiting its potential. Keyword extraction assists in making better sense of these vast information and data resources and leverage their value. In this article we propose and analyze keyword extraction methodology using Rényi entropy and Tsallis relative entropy. The proposed methods being statistics-based methods, these are language and domain independent method. The proposed methodology may find applications in dynamic text collection, information retrieval, natural language processing etc.
\end{abstract}

Keywords: Text mining; Keyword extraction; Rényi entropy; Tsallis relative entropy; Dynamic text collection.

\section{INTRODUCTION}

Keyword extraction plays a significant role in text mining. Text mining aims at extricating high-quality information from the given pool of textual data. In simpler terms it is the process of identifying key or relevant terms from a document, that supports in representing an suitable subject of the text. Huge quantity of new data and text is being generated through various social, economic and academic activities, with a substantial potential economic and social value. It is the techniques like text and data mining that assists in exploiting this potential. Text mining aids in assimilating, analyzing, interpreting the unstructured pool of data and puts the inferences for the apt and prompt use. Organizing and maintaining this dynamic text collection is challenging, time-consuming, expensive and tedious. And this is where keyword extraction, especially domain independent statistical keyword extraction plays an important role by helping in determining the relevant documents from a large pool of available data (Rossi, Marcacini and Rezende, 2014).

Keyword extraction has applications in text mining, information retrieval, web page retrieval, natural language processing, incremental clustering applications (Beliga, 2014; Rossi, Marcacini and Rezende, 2014) . Various methodologies for keyword extraction are broadly classified as: Linguistic, Machine learning and Statistical (Beliga, 2014). Linguistic approaches are usually derived from the linguistic attributes and are based on syntactic and semantic structures in the text. The linguistic approach comprises of the lexical analysis, syntactic analysis, discourse analysis and so on (Siddiqi and Sharan, 2015). Machine Learning approaches normally consider supervised learning methods. In these methods a model is trained based on a set of keywords extracted from training documents, and then the model is tested for performance through a testing module. Based on performance evaluation a satisfactory model is chosen for keyword extraction from new documents. This approach uses Naïve Bayes, Support Vector Machine, etc (Beliga, 2014; Jamaati and Mehri, 2018). Statistical methods require neither the training data nor the prior domain knowledge. Additionally, the statistical methods are language independent. In these methods the statistics of the words from the document forms a basis for keyword identification. The statistical methods are based on the following characteristics of text like term frequency (Luhn, 1958), standard deviation (Ortuno et al., 2002), 
centrality measure, spatial distribution (Carpena et al., 2009), entropy (Herrera and Pury, 2008), word co-occurrences (Matsuo and Ishizuka, 2004), etc. The statistical methods are computationally more efficient than other methods but sometimes they may exhibit lower accuracy for some health and medical texts where the most substantial keywords may have low frequency. Statistical models may inadvertently not consider such words as keywords (Chen and Lin, 2010).

\section{ENTROPY}

Entropy in general is a measure of uncertainty or randomness of a system. C.E. Shannon (Shannon, 1948) introduced the concept of entropy to information theory. Let $\mathrm{X}$ be a discrete random variable with possible values $\left\{\mathrm{x}_{1}, \mathrm{x}_{2}, \ldots \mathrm{x}_{\mathrm{n}}\right\}$ and probability mass function $\mathrm{P}_{\mathrm{d}}(\mathrm{X})=\left\{\mathrm{p}_{1}, \mathrm{p}_{2}, \ldots, \mathrm{p}_{\mathrm{n}}\right\}$, then the Shannon's entropy (Shannon, 1948 ) is given by

$$
\mathrm{S}_{\mathrm{S}}\left(\mathrm{P}_{\mathrm{d}}\right)=-\sum_{\mathrm{i}}^{\mathrm{N}}=1 \mathrm{p}_{\mathrm{i}} \log \mathrm{p}_{\mathrm{i}}
$$

Using the exponential mean, Rényi proposed a new generalized information measure commonly termed as Rényi’s information measure or Rényi's entropy (Rényi and others, 1961):

$$
\mathrm{S}_{\mathrm{R}}\left(\mathrm{P}_{\mathrm{d}}, \mathrm{q}\right)=\frac{1}{(1-\mathrm{q})} \log _{\mathrm{b}} \Sigma_{\mathrm{i}}^{\mathrm{N}}=1 \mathrm{p}_{\mathrm{i}}^{\mathrm{q}}
$$

where $\mathrm{b}$ is the logarithm base and $\mathrm{q}>0$ is a real parameter.

Considering applications to non-extensive systems, Tsallis (Tsallis, 1988) proposed a generalized entropy given by:

$$
\mathrm{S}_{\mathrm{T}}\left(\mathrm{P}_{\mathrm{d}}, \mathrm{q}\right)=\frac{1}{(1-\mathrm{q})}\left(\sum_{\mathrm{i}}^{\mathrm{N}}=1 \mathrm{p}_{\mathrm{i}}^{\mathrm{q}}-1\right)
$$

As $\rightarrow 1$, both Rényi's $S_{R}\left(P_{d}, q\right)$ and Tsallis entropy $S_{R}\left(P_{d}, q\right)$ approaches Shannon's entropy

$$
S_{S}\left(P_{d}\right) \text { i.e. } S_{R}\left(P_{d}, 1\right)=S_{S}\left(P_{d}\right) \text {. }
$$

In information theory, The (Kullback and Leibler, 1951) relative entropy is a measure of how one probability distribution varies or differs from another distribution and is given by

$$
D_{K L}(P \| Q)=\sum_{i}{ }^{n}=1 p_{i} \log \left(\frac{p i}{q i}\right)
$$

Tsallis relative entropy (Furuichi, Shigeru, Kenjiro Yanagi, 2004) is a generalization to relative entropy and is given by:

$$
\mathrm{D}_{\text {Tsallis }}(\mathrm{P} \| \mathrm{Q})=-\sum_{\mathrm{i}}{ }^{\mathrm{n}}=1 \mathrm{p}_{\mathrm{i}} \ln _{\mathrm{q}}\left(\frac{\mathrm{pi}}{\mathrm{qi}}\right)
$$

where $\ln _{\mathrm{q}}(\mathrm{x})=\left(\mathrm{x}^{1-\mathrm{q}-1}\right) / 1-\mathrm{q}, \mathrm{q} \neq 1$. As $\mathrm{q} \rightarrow 1, \ln _{\mathrm{q}}(\mathrm{x}) \rightarrow \ln (\mathrm{x})$ and $\mathrm{D}_{\text {Tsallis }}(\mathrm{P} \| \mathrm{Q}) \rightarrow \mathrm{D}_{\mathrm{KL}}(\mathrm{P} \| \mathrm{Q})$.

Keyword extraction using Tsallis entropy has been explored in (Jamaati and Mehri, 2018). Rényi entropy has been successful in exploiting multifractal systems (Jizba and Arimitsu, 2004) and systems with mixed population of random variable. Moreover, Rényi entropy has successful application in the complex systems (Bashkirov, 2006) and the fact that the maximum entropy principle results in the same form of q-exponential distribution function for both Tsallis (Tsallis, 1988) and Rényi entropies (Johal and Tirnakli, 2004), we aim at exploring the use of Rényi entropy for keyword extraction. We have also explored the keyword extraction methodology using Tsallis relative entropy in this manuscript. 


\section{METHODOLOGY}

This section aims at defining the methodology used for extrication relevant words from the given text using Rényi entropy and Tsallis relative entropy. The various notations used in the paper are as follows:

Table 1. List of common notations used in methodology for extracting relevant words from a given text

\begin{tabular}{|c|c|}
\hline Notation & Description \\
\hline $\mathrm{N}$ & Difference between the positions of last and first occurrences of word $\mathrm{w}$ \\
\hline $\mathrm{d}_{\mathrm{i}}(\mathrm{w})$ & Distance between $\mathrm{i}^{\text {th }}$ and $(\mathrm{i}+1)^{\text {th }}$ occurrences of word $\mathrm{w}$ \\
\hline $\mathrm{p}_{\mathrm{i}}(\mathrm{w})$ & $\begin{array}{l}\text { Probability distribution is obtained by dividing } \mathrm{d}_{\mathrm{i}}(\mathrm{w}) \text {, the distance } \\
\text { between } \mathrm{i}^{\text {th }} \text { and }(\mathrm{i}+1)^{\text {th }} \text { occurrences of word } \mathrm{w} \text { by } \mathrm{N}\end{array}$ \\
\hline $\mathrm{F}_{\mathrm{w}}$ & Frequency of intended word w \\
\hline $\mathrm{R}(\mathrm{w}, \mathrm{q})$ & Rényi entropy-based word ranking parameter \\
\hline$\Delta \mathrm{S}_{\mathrm{T}}(\mathrm{w}, \mathrm{q})$ & Tsallis entropy-based word ranking measure [(Jamaati and Mehri, 2018)] \\
\hline $\mathrm{D}_{\text {Tsallis }}(\mathrm{P} \| \mathrm{Q})$ & Tsallis relative entropy \\
\hline
\end{tabular}

To start with, a probability distribution is obtained as $\mathrm{p}_{\mathrm{i}}(\mathrm{w})=\mathrm{d}_{\mathrm{i}}(\mathrm{w}) / \mathrm{N}$. The probability distribution satisfies the normalization condition: $\sum_{\mathrm{i}}{ }^{\mathrm{FW}}=1 \mathrm{p}_{\mathrm{i}}(\mathrm{W})=1$ as $\sum_{\mathrm{i}}{ }^{\mathrm{FW}}=1 \mathrm{~d}_{\mathrm{i}}(\mathrm{W})=\mathrm{N}$. Rényi entropy-based word ranking measure is evaluated for all word types in the given text and a word ranking measure using Rényi entropy is defined as:

$$
R(w, q)=\left|\log _{2} F_{w}-S_{R}(w, q)\right|
$$

where $\mathrm{S}_{\mathrm{R}}(\mathrm{w}, \mathrm{q})=\frac{1}{(1-\mathrm{q})} \log _{2} \sum_{\mathrm{i}}^{\mathrm{FW}}=1 \mathrm{p}_{\mathrm{i}}^{\mathrm{q}}$. For even distribution of the word type $\mathrm{w}$ in the text and $0<\mathrm{q} \leq 2$ and $\mathrm{q} \neq 1, \mathrm{~S}_{\mathrm{R}}$ $(\mathrm{w}, \mathrm{q})$ approaches $\log _{2} \mathrm{~N}_{\mathrm{w}}$. It is observed that grammatical words are more homogeneously distributed in the text, and therefore have lower values for the word relevance ranking parameter $\mathrm{R}(\mathrm{w}, \mathrm{q})$. In contrast, the relevant words are not so evenly spread and hence have greater values for parameter $\mathrm{R}(\mathrm{w}, \mathrm{q})$. Words are sorted in descending order of $\mathrm{R}(\mathrm{w}, \mathrm{q})$ value. The most relevant words appear in the upper part of the sorted list, which can be considered as the retrieved set or index.

Since grammatical words are more homogeneously spread in the text, they should have lower values of relative entropies when evaluated with respect to a completely homogeneous distribution. The homogeneous probability distribution is obtained as $\mathrm{q}_{\mathrm{i}}(\mathrm{w})=\frac{1}{\mathrm{~F}_{\mathrm{w}}}$. The methodology for extracting relevant words from a given text based on Tsallis, Rényi entropy and Tsallis relative entropy are as shown in Figure 1 and Figure 2 respectively. 
Evaluate di(w)) and FW for distinct words in the text.

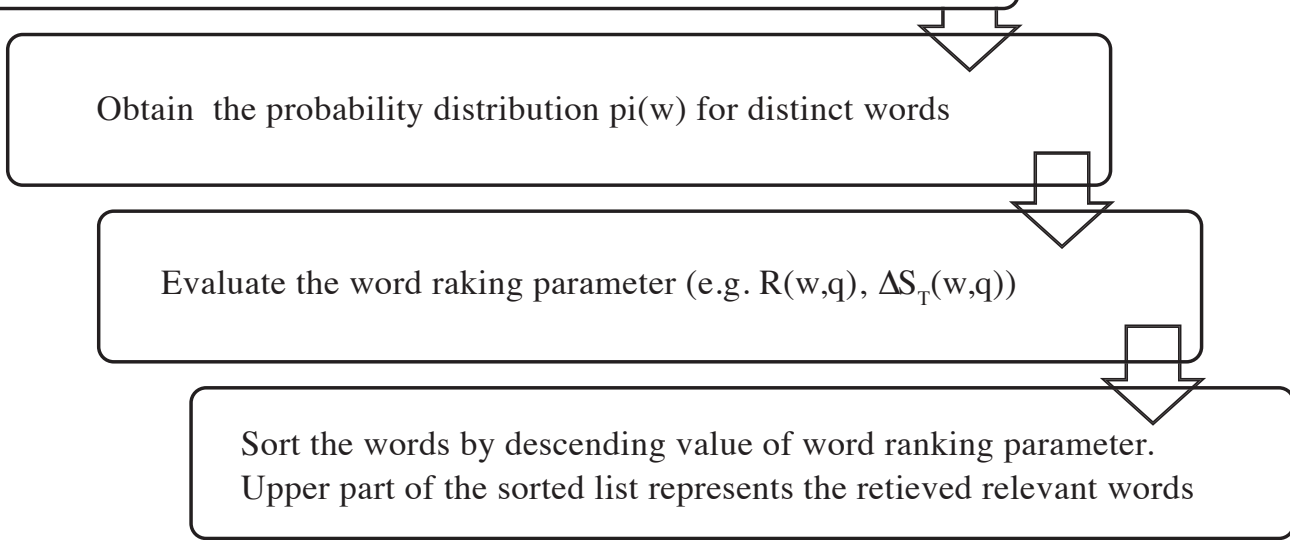

Figure 1. Methodology for extracting retrieved list from given text using entropy

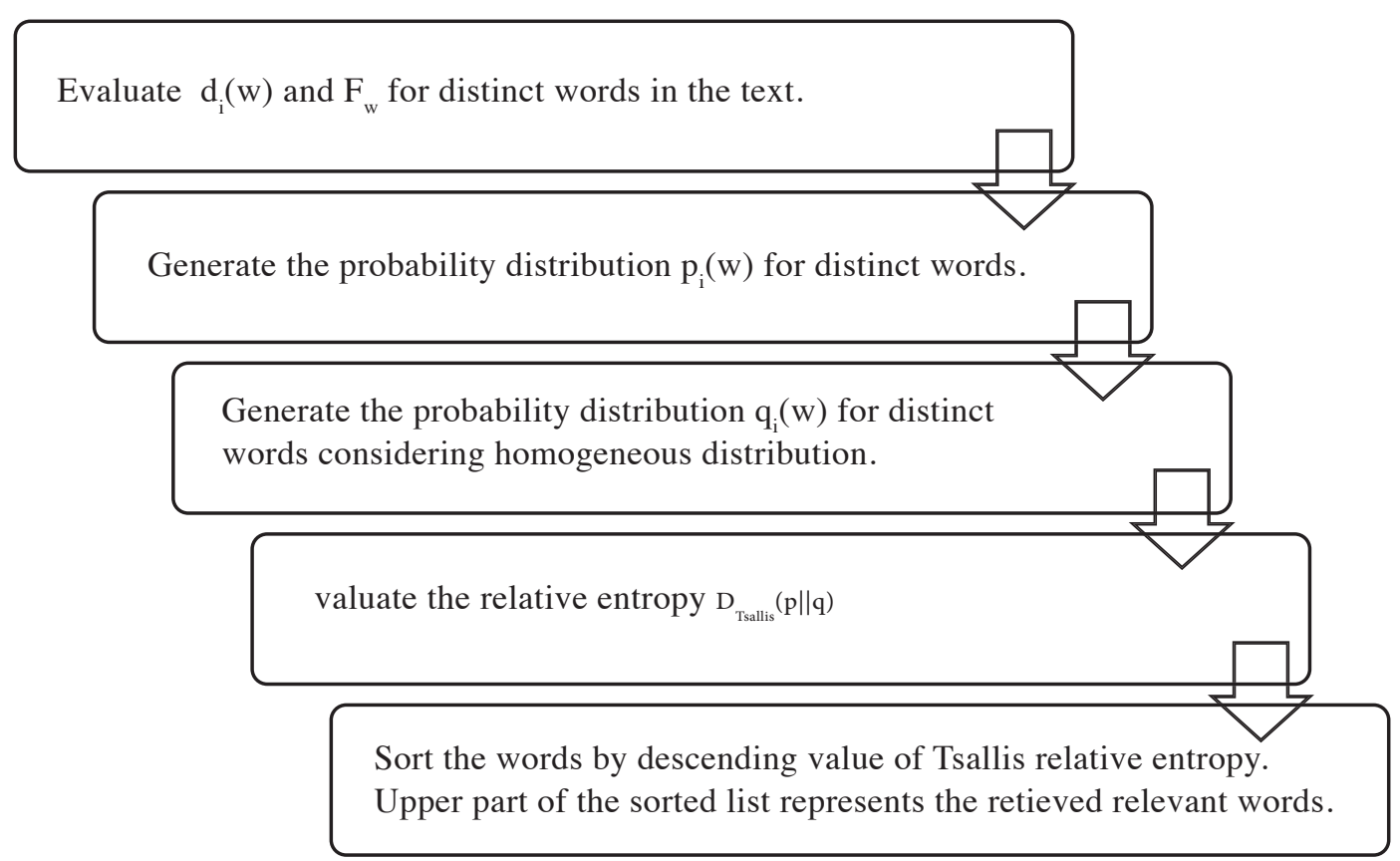

Figure 2. Methodology for extracting retrieved list from given text using Tsallis relative entropy 


\section{RESULTS AND DISCUSSIONS}

Both the actual and relative performance of proposed Rényi entropy-based keyword extraction method has been shown. The relative performance of Rényi entropy-based word ranking parameter has been evaluated with respect to Tsallis entropy-based word ranking parameter [4]. The books used for evaluating the performance of proposed word ranking method are: The essence of Hinduism (Gandhi, 1987)(Book 1) and Global warming: the complete briefing (Houghton, 2009) (Book 2). Firstly, the books are obtained as text files. Post deleting the punctuations \& numerals and converting the complete text to alphabetical lower case, space character in text is used as a separator to extricate consecutive terms as individual words. A set of relevant words have been extracted from the index of given books. The cardinality of this relevant set is 244 and 551 for Book 1 and Book 2 respectively. These sets are used to evaluate the actual performance of proposed word ranking metric.

Since no stemming and lemmatization process have been applied as pre-processes, all inflectional and derivationally related forms of a word are considered as different words.

Let $\mathrm{N}_{\text {rel }}$ and $\mathrm{N}_{\text {ret }}$ respectively represent the cardinality of relevant and retrieved sets and $\mathrm{N}_{\text {rel } \cap \text { ret }}$ denote the cardinality of the relevant and retrieved sets, then recall $\mathrm{R}$, precision $\mathrm{P}$ and $\mathrm{f}$-measure $\mathrm{F}$ are given by:

$R=\frac{N_{\text {rellnet }}}{N_{\text {rel }}}, P=\frac{N_{\text {rell }} \text { ret }}{N_{\text {rel }}}, F=\frac{2 R P}{R+P}$

The above measures have been calculated and analysed considering different sets as relevant and retrieved as mentioned in Table 2.

Table 2. Recall (R), Precision(P) and f-measure for Book 1 and Book 2

\begin{tabular}{|c|c|c|c|}
\hline & Step 1 & Step 2 & Step 3 \\
\hline Relevant set & Generated using index & Generated using index & $\begin{array}{c}\text { Extracted using } \\
\Delta S T(w, 0.8)\end{array}$ \\
\hline Retrieved set & $\begin{array}{c}\text { Extracted using } \\
\Delta S \_T(w, 0.8)\end{array}$ & Extracted using $R(w, 2)$ & Extracted using $R(w, 2)$ \\
\hline
\end{tabular}

\begin{tabular}{|c|c|c|c|c|c|c|}
\hline Book & 1 & 2 & 1 & 2 & 1 & 2 \\
\hline N_rel & 244 & 551 & 244 & 551 & 244 & 551 \\
\hline N_ret & 244 & 551 & 244 & 551 & 244 & 551 \\
\hline N_(rel $\cap$ ret) & 60 & 222 & 60 & 201 & 188 & 458 \\
\hline R & 0.2459 & 0.4029 & 0.2459 & 0.3648 & 0.7705 & 0.8312 \\
\hline P & 0.2459 & 0.4029 & 0.2459 & 0.3648 & 0.7705 & 0.8312 \\
\hline F & 0.2459 & 0.4029 & 0.2459 & 0.3648 & 0.7705 & 0.8312 \\
\hline
\end{tabular}


Table 3 and Table 4 displays the list of top 20 words extracted from Book 1 and Book 2 respectively using entropy-based keyword extraction methodology. Words have been categorized as relevant based on their existence in the respective indexes. It is interesting to note that some words like hate, adhikara, son, dasharatha, in Book 1 are indicated as irrelevant in Table 3 as per their unavailability in Index, but are essentially not irrelevant and seem relevant to Book 1. These words being quite familiar might not have been included in the index. Likewise, few terms like commercial, God, cost, wec (abbreviation of World Energy Council), Cambridge are classified as irrelevant in Table 4, but appears pertinent in accordance with comprehensive text in Book 2. This unveils the exactitude of the defined keyword extraction methodology using Rényi entropy.

Table 3. Top 20 words retrieved from Book 1 based on keyword extraction methodology using entropy

\begin{tabular}{|c|c|c|c|c|c|c|c|c|}
\hline \multirow{2}{*}{$\begin{array}{c}\frac{\bar{z}}{0} \\
\text { 递 } \\
\text { Rank }\end{array}$} & \multicolumn{4}{|c|}{$\begin{array}{l}\text { Top } 20 \text { words using methodology based } \\
\text { on Rényi entropy }\end{array}$} & \multicolumn{4}{|c|}{$\begin{array}{l}\text { Top } 20 \text { words using methodology based } \\
\text { on Tsallis entropy }\end{array}$} \\
\hline & Word & Frequency & $\mathbf{R}(\mathbf{w}, 2)$ & Relevance & Words & Frequency & $\Delta S_{\mathrm{T}}(\mathrm{w}, \mathbf{0 . 8})$ & Relevance \\
\hline 1 & prayer & 293 & 4.452 & Relevant & prayer & 293 & 4.457 & Relevant \\
\hline 2 & congregational & 27 & 3.967 & Relevant & gita & 227 & 3.943 & Relevant \\
\hline 3 & gita & 227 & 3.915 & Relevant & ramanama & 94 & 3.880 & Relevant \\
\hline 4 & renunciation & 31 & 3.872 & Relevant & hinduism & 228 & 3.826 & Relevant \\
\hline 5 & mantra & 38 & 3.858 & Relevant & renunciation & 31 & 3.644 & Relevant \\
\hline 6 & ramanama & 94 & 3.700 & Relevant & mantra & 38 & 3.603 & Relevant \\
\hline 7 & son & 13 & 3.675 & Irrelevant & cow & 31 & 3.374 & Relevant \\
\hline 8 & hinduism & 228 & 3.674 & Relevant & ashram & 58 & 3.250 & Relevant \\
\hline 9 & dasharatha & 12 & 3.559 & Irrelevant & hate & 24 & 3.215 & Irrelevant \\
\hline 10 & maths & 12 & 3.527 & Relevant & you & 425 & 3.213 & Irrelevant \\
\hline 11 & cow & 31 & 3.470 & Relevant & son & 13 & 3.207 & Irrelevant \\
\hline 12 & vows & 15 & 3.380 & Relevant & rama & 65 & 3.154 & Relevant \\
\hline 13 & ashram & 58 & 3.379 & Relevant & congregational & 27 & 3.102 & Relevant \\
\hline 14 & ishopanishad & 14 & 3.349 & Relevant & dasharatha & 12 & 3.078 & Irrelevant \\
\hline 15 & rama & 65 & 3.346 & Relevant & god & 674 & 3.002 & Relevant \\
\hline 16 & hate & 24 & 3.313 & Irrelevant & maths & 12 & 2.982 & Relevant \\
\hline 17 & sanatani & 13 & 3.204 & Relevant & students & 36 & 2.936 & Relevant \\
\hline 18 & students & 36 & 3.151 & Relevant & he & 589 & 2.898 & Irrelevant \\
\hline 19 & englishmen & 9 & 3.136 & Relevant & $\mathrm{i}$ & 1161 & 2.873 & Irrelevant \\
\hline 20 & adhikara & 9 & 3.115 & Irrelevant & evil & 69 & 2.822 & Relevant \\
\hline
\end{tabular}




\begin{tabular}{|c|c|c|c|c|c|c|c|c|}
\hline \multirow{2}{*}{$\begin{array}{c}\frac{N}{8} \\
0 \\
0 \\
\text { Rank }\end{array}$} & \multicolumn{4}{|c|}{$\begin{array}{l}\text { Top } 20 \text { words using methodology based } \\
\text { on Rényi entropy }\end{array}$} & \multicolumn{4}{|c|}{$\begin{array}{l}\text { Top } 20 \text { words using methodology based } \\
\text { on Rényi entropy }\end{array}$} \\
\hline & Word & Frequency & $\mathbf{R}(\mathbf{w}, 2)$ & Relevance & Words & Frequency & $\Delta S_{T}(w, 0.8)$ & Relevance \\
\hline 1 & stabilization & 5.048 & 84 & Relevant & $\mathrm{j}$ & 5.189 & 4256 & Irrelevant \\
\hline 2 & cost & 4.937 & 185 & Irrelevant & cambridge & 4.910 & 204 & Irrelevant \\
\hline 3 & god & 4.412 & 24 & Irrelevant & energy & 4.755 & 666 & Relevant \\
\hline 4 & ice & 4.368 & 162 & Relevant & stabilization & 4.431 & 84 & Relevant \\
\hline 5 & renewable & 4.282 & 105 & Relevant & $\mathrm{pp}$ & 4.261 & 125 & Irrelevant \\
\hline 6 & sustainable & 4.119 & 61 & Relevant & cost & 4.187 & 185 & Irrelevant \\
\hline 7 & mitigation & 4.052 & 68 & Relevant & et & 4.144 & 158 & Irrelevant \\
\hline 8 & commercial & 4.041 & 25 & Irrelevant & radiation & 4.034 & 175 & Relevant \\
\hline 9 & cloud & 4.023 & 56 & Relevant & ice & 4.025 & 162 & Relevant \\
\hline 10 & $\mathrm{j}$ & 3.988 & 256 & Irrelevant & cloud & 3.921 & 56 & Relevant \\
\hline 11 & wec & 3.947 & 33 & Irrelevant & god & 3.888 & 24 & Irrelevant \\
\hline 12 & cambridge & 3.938 & 204 & Irrelevant & al & 3.857 & 165 & Irrelevant \\
\hline 13 & environment & 3.902 & 111 & Relevant & renewable & 3.813 & 105 & Relevant \\
\hline 14 & runoff & 3.892 & 21 & Relevant & carbon & 3.677 & 631 & Relevant \\
\hline 15 & costs & 3.864 & 89 & Irrelevant & press & 3.549 & 140 & Irrelevant \\
\hline 16 & savings & 3.847 & 33 & Relevant & $\mathrm{ppm}$ & 3.492 & 75 & Irrelevant \\
\hline 17 & energy & 3.844 & 666 & Relevant & ocean & 3.476 & 215 & Relevant \\
\hline 18 & cores & 3.843 & 20 & Relevant & climate & 3.469 & 1162 & Relevant \\
\hline 19 & radiative & 3.822 & 86 & Relevant & religious & 3.459 & 28 & Irrelevant \\
\hline 20 & particles & 3.800 & 39 & Relevant & forcing & 3.448 & 100 & Relevant \\
\hline
\end{tabular}
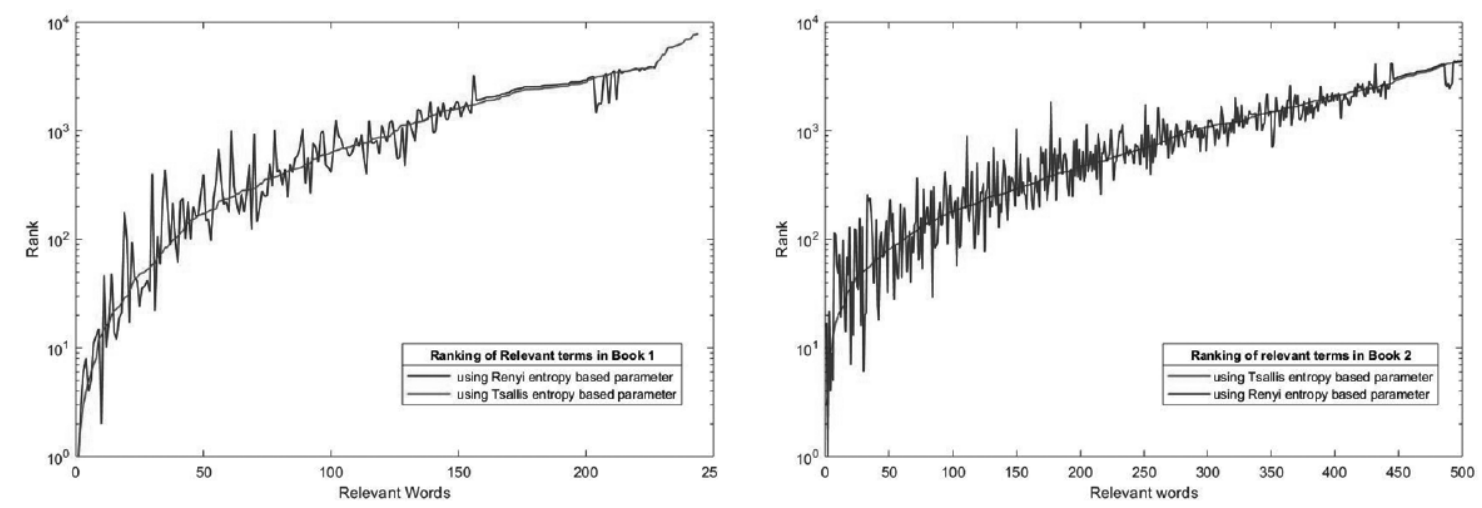

Figure 3. Shows the comparison of word rankings using Rényi and Tsallis entropy-based parameter for relevant words from Book 1 and Book 2. 
Figure 3 shows the comparisons of word ranking using Rényi and Tsallis entropy-based parameter for relevant words from Book 1 and Book 2. As visible in Figure 3 the ranks of relevant words are closely related. The correlation coefficient between word ranking using parameter based on Rényi and Tsallis entropy are 0.9850 and 0.9779 for Book 1 and Book 2 respectively, which indicate a strong statistical association between the two variables.

Further, we have used the Tsallis relative entropy for extracting keywords from given text using the methodology based on relative entropy. Since Tsallis relative entropy is a generalization to Kullback \& Leibler divergence, for entropic index $\mathrm{q}=1$, results correspond to Kullback \& Leibler divergence. Figure 5 exhibits the Tsallis relative entropy values for various entropic index values ranging from 0.2 to 4.0 for top 30 words retrieved from Book 1 using methodology based on Tsallis relative entropy for entropic index $q=3$. The entropic index can be chosen based on the non-extensivity of the system. To improve the accuracy of keyword extraction process machine learning approach may be employed for deciding on the value of entropic index. Figure 6 shows the variation of Tsallis relative entropy values for varying values of entropic index q for top 30 words retrieved from Book 1 using methodology based on Tsallis relative entropy for entropic index $q=3$. It can be observed that higher values of entropic index $q$ provides more leverage to words with higher frequencies.

\begin{tabular}{|c|c|c|c|c|c|c|c|c|c|c|c|c|}
\hline String & Frequency & $q=0.2$ & $q=0.6$ & $q=1$ & $q=1.4$ & $q=1.8$ & $q=2.2$ & $q=2.6$ & $q=3$ & $q=3.4$ & $q=3.8$ & $q=4$ \\
\hline maths & 12 & 1.042 & 1.504 & 2.331 & 3.893 & 6.986 & 13.354 & 26.902 & 56.491 & 122.517 & 272.411 & 409.276 \\
\hline hate & 24 & 0.570 & 0.834 & 1.318 & 2.263 & 4.203 & 8.360 & 17.587 & 38.646 & 87.801 & 204.619 & 314.771 \\
\hline definitions & 15 & 0.760 & 1.088 & 1.667 & 2.743 & 4.829 & 9.032 & 17.766 & 36.384 & 76.907 & 166.606 & 247.059 \\
\hline atheists & 15 & 0.721 & 1.027 & 1.562 & 2.544 & 4.422 & 8.148 & 15.768 & 31.746 & 65.934 & 140.312 & 206.211 \\
\hline thee & 11 & 0.872 & 1.224 & 1.822 & 2.883 & 4.836 & 8.553 & 15.827 & 30.395 & 60.128 & 121.783 & 174.579 \\
\hline englishmen & 9 & 0.999 & 1.390 & 2.043 & 3.179 & 5.224 & 9.022 & 16.267 & 30.393 & 58.443 & 114.998 & 162.469 \\
\hline dedication & 10 & 0.922 & 1.289 & 1.904 & 2.984 & 4.948 & 8.635 & 15.745 & 29.770 & 57.955 & 115.477 & 164.182 \\
\hline atheist & 13 & 0.715 & 1.002 & 1.489 & 2.348 & 3.924 & 6.910 & 12.725 & 24.314 & 47.850 & 96.403 & 137.827 \\
\hline wealth & 9 & 0.901 & 1.240 & 1.796 & 2.739 & 4.394 & 7.384 & 12.917 & 23.372 & 43.476 & 82.702 & 114.864 \\
\hline renunciation & 31 & 0.399 & 0.579 & 0.903 & 1.521 & 2.760 & 5.344 & 10.917 & 23.263 & 51.217 & 115.625 & 175.052 \\
\hline conditions & 14 & 0.667 & 0.935 & 1.391 & 2.198 & 3.681 & 6.500 & 12.005 & 23.008 & 45.424 & 91.809 & 131.472 \\
\hline cow & 31 & 0.392 & 0.567 & 0.881 & 1.480 & 2.672 & 5.145 & 10.446 & 22.118 & 48.378 & 108.496 & 163.713 \\
\hline bihar & 13 & 0.673 & 0.938 & 1.381 & 2.153 & 3.547 & 6.144 & 11.114 & 20.835 & 40.206 & 79.399 & 112.380 \\
\hline shlokas & 8 & 0.863 & 1.167 & 1.648 & 2.434 & 3.755 & 6.031 & 10.041 & 17.238 & 30.361 & 54.616 & 73.743 \\
\hline blazer & 7 & 0.938 & 1.258 & 1.759 & 2.564 & 3.895 & 6.147 & 10.037 & 16.878 & 29.092 & 51.186 & 68.341 \\
\hline ambedkar & 7 & 0.938 & 1.258 & 1.758 & 2.564 & 3.894 & 6.145 & 10.033 & 16.869 & 29.074 & 51.151 & 68.293 \\
\hline habits & 7 & 0.935 & 1.254 & 1.752 & 2.552 & 3.874 & 6.109 & 9.966 & 16.742 & 28.829 & 50.672 & 67.621 \\
\hline vegetarianism & 7 & 0.934 & 1.253 & 1.750 & 2.550 & 3.870 & 6.100 & 9.950 & 16.711 & 28.769 & 50.555 & 67.456 \\
\hline enjoyment & 7 & 0.933 & 1.251 & 1.747 & 2.545 & 3.861 & 6.084 & 9.920 & 16.654 & 28.659 & 50.340 & 67.155 \\
\hline deny & 11 & 0.683 & 0.935 & 1.343 & 2.028 & 3.215 & 5.327 & 9.176 & 16.335 & 29.875 & 55.854 & 76.901 \\
\hline ahimsa & 39 & 0.288 & 0.414 & 0.638 & 1.056 & 1.872 & 3.533 & 7.015 & 14.511 & 30.987 & 67.828 & 101.108 \\
\hline thy & 7 & 0.873 & 1.163 & 1.609 & 2.316 & 3.465 & 5.375 & 8.610 & 14.186 & 23.939 & 41.210 & 54.417 \\
\hline creed & 10 & 0.662 & 0.894 & 1.260 & 1.856 & 2.855 & 4.570 & 7.580 & 12.960 & 22.730 & 40.713 & 54.852 \\
\hline untouchability & 33 & 0.306 & 0.435 & 0.659 & 1.067 & 1.842 & 3.366 & 6.456 & 12.876 & 26.484 & 55.806 & 81.609 \\
\hline quetta & 9 & 0.688 & 0.922 & 1.284 & 1.865 & 2.820 & 4.428 & 7.188 & 12.012 & 20.572 & 35.957 & 47.849 \\
\hline congress & 10 & 0.637 & 0.856 & 1.200 & 1.755 & 2.676 & 4.242 & 6.961 & 11.767 & 20.395 & 36.087 & 48.320 \\
\hline relief & 6 & 0.889 & 1.163 & 1.573 & 2.201 & 3.184 & 4.752 & 7.297 & 11.490 & 18.492 & 30.319 & 39.057 \\
\hline renounce & 6 & 0.881 & 1.151 & 1.555 & 2.173 & 3.138 & 4.674 & 7.160 & 11.248 & 18.057 & 29.528 & 37.987 \\
\hline puranas & 7 & 0.790 & 1.041 & 1.421 & 2.010 & 2.946 & 4.463 & 6.967 & 11.164 & 18.301 & 30.579 & 39.773 \\
\hline temples & 12 & 0.545 & 0.736 & 1.038 & 1.530 & 2.356 & 3.777 & 6.274 & 10.744 & 18.875 & 33.864 & 45.663 \\
\hline
\end{tabular}

Figure 5. Frequency and Tsallis relative entropy values of top 30 words extracted from Book 1 using relative Tsallis entropy-based methodology for entropic index $q=3$ 


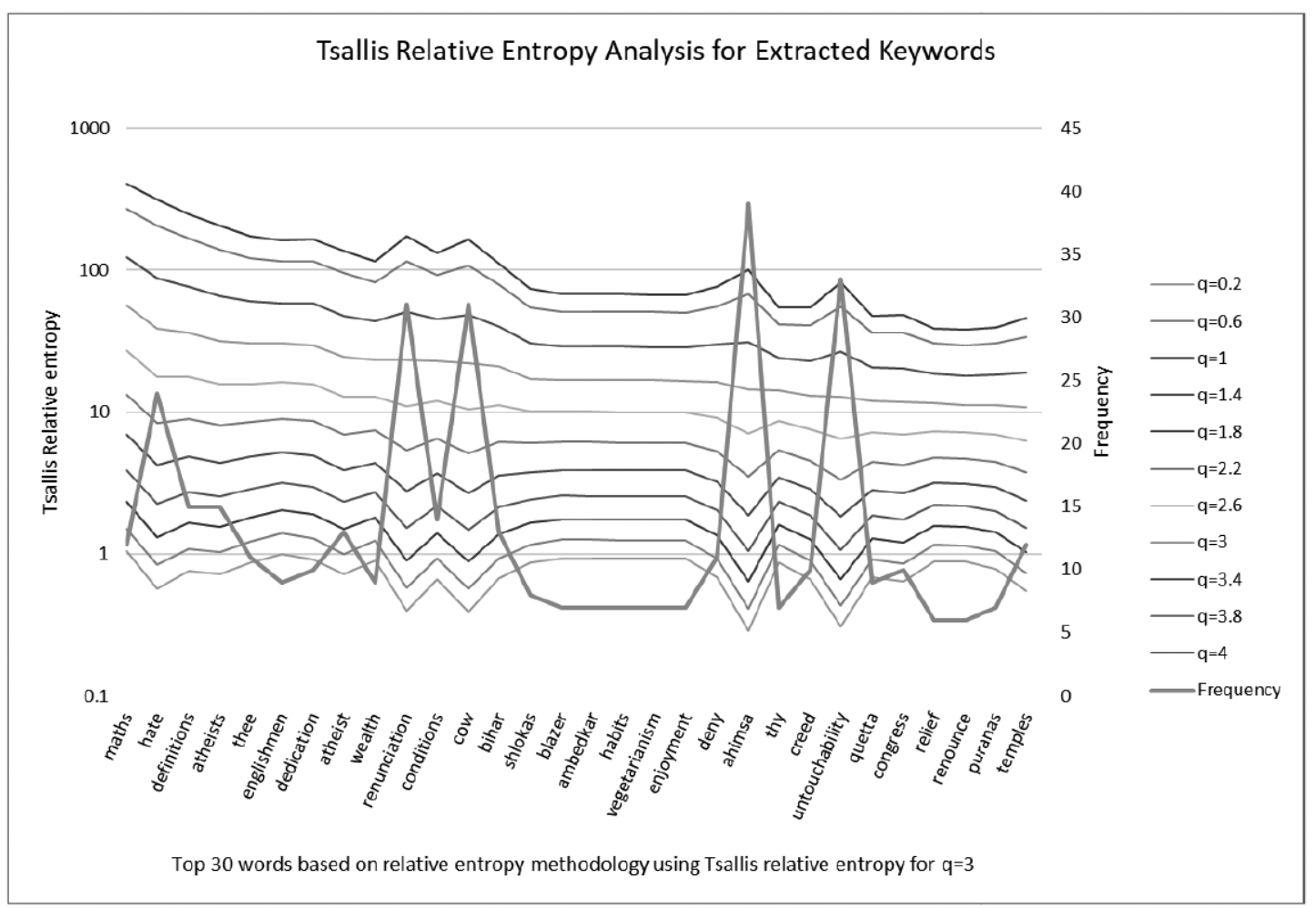

Figure 6. Shows the variation of Tsallis relative entropy values for varying values of entropic index $q$ for top 30 words retrieved from Book 1 using methodology based on Tsallis relative entropy for entropic index $q=3$.

\section{CONCLUSION}

The proposed Rényi entropy and Tsallis relative entropy-based methodologies are successful in extricating pertinent words from the text. It is interesting to note that proposed methodology was effective in even extracting the terms that being quite common and familiar were missing in the index or glossary but were pertinent to the text. A reliable performance in keyword extraction was achieved using entropic index of $q=2$ for Rényi entropy and $q=3$ for Tsallis relative entropy-based methodology. Better results can be obtained by using lemmatization and stemming as pre-processing tools. Being a statistical method, proposed keyword extraction method are domain and language independent and therefore may have applications in organizing dynamic text collection, incremental clustering applications and many related applications. Also, for improvised performance machine learning approaches can be employed to evaluate the value of entropic index best suitable for the text.

\section{REFERENCES}

Bashkirov, A. G. 2006. Renyi entropy as a statistical entropy for complex systems, Theoretical and Mathematical Physics, 149(2), pp. 1559-1573.

Beliga, S. 2014. Keyword extraction: a review of methods and approaches, University of Rijeka, Department of Informatics, pp. 1-9.

Carpena, P. et al. 2009. Level statistics of words: Finding keywords in literary texts and symbolic sequences, Physical Review E, 79(3), p. 35102.

Chen, P.-I. and Lin, S.-J. 2010. Automatic keyword prediction using Google similarity distance, Expert Systems with Applications, 37(3), pp. 1928-1938. 
Furuichi, Shigeru, Kenjiro Yanagi, and K. K. 2004. Fundamental properties of Tsallis relative entropy., Journal of Mathematical Physics, 45(12), pp. 4868-4877.

Gandhi, M. 1987. The essence of Hinduism. Navajivan Publishing House Ahmedabad.

Herrera, J. P. and Pury, P. A. 2008. Statistical keyword detection in literary corpora, The European Physical Journal B, 63(1), pp. 135-146.

Houghton, J. 2009. Global warming: the complete briefing. Cambridge university press.

Jamaati, M. and Mehri, A. 2018. Text mining by Tsallis entropy, Physica A: Statistical Mechanics and its Applications, 490, pp. 1368-1376.

Jizba, P. and Arimitsu, T. 2004. The world according to Rényi: thermodynamics of multifractal systems, Annals of Physics, 312(1), pp. 17-59.

Johal, R. S. and Tirnakli, U. 2004. Tsallis versus Renyi entropic form for systems with q-exponential behaviour: the case of dissipative maps, Physica A: Statistical Mechanics and its Applications, 331(3-4), pp. 487-496.

Kullback, S. and Leibler, R. A. 1951. On information and sufficiency, The annals of mathematical statistics, 22(1), pp. 79-86.

Bashkirov, A. G. 2006. Renyi entropy as a statistical entropy for complex systems, Theoretical and Mathematical Physics, 149(2), pp. 1559-1573.

Beliga, S. 2014. Keyword extraction: a review of methods and approaches, University of Rijeka, Department of Informatics, pp. 1-9.

Carpena, P. et al. 2009. Level statistics of words: Finding keywords in literary texts and symbolic sequences, Physical Review E, 79(3), p. 35102.

Chen, P.-I. and Lin, S.-J. 2010. Automatic keyword prediction using Google similarity distance, Expert Systems with Applications, 37(3), pp. 1928-1938.

Furuichi, Shigeru, Kenjiro Yanagi, and K. K. 2004. Fundamental properties of Tsallis relative entropy., Journal of Mathematical Physics, 45(12), pp. 4868-4877.

Gandhi, M. 1987. The essence of Hinduism. Navajivan Publishing House Ahmedabad.

Herrera, J. P. and Pury, P. A. 2008. Statistical keyword detection in literary corpora, The European Physical Journal B, 63(1), pp. 135-146.

Houghton, J. 2009. Global warming: the complete briefing. Cambridge university press.

Jamaati, M. and Mehri, A. 2018. Text mining by Tsallis entropy, Physica A: Statistical Mechanics and its Applications, 490, pp. 1368-1376.

Jizba, P. and Arimitsu, T. 2004. The world according to Rényi: thermodynamics of multifractal systems, Annals of Physics, 312(1), pp. 17-59.

Johal, R. S. and Tirnakli, U. 2004. Tsallis versus Renyi entropic form for systems with q-exponential behaviour: the case of dissipative maps, Physica A: Statistical Mechanics and its Applications, 331(3-4), pp. 487-496.

Kullback, S. and Leibler, R. A. 1951. On information and sufficiency, The annals of mathematical statistics, 22(1), pp. 79-86.

Luhn, H. P. 1958. The automatic creation of literature abstracts, IBM Journal of research and development, 2(2), pp. 159-165. 
Matsuo, Y. and Ishizuka, M. 2004. Keyword extraction from a single document using word co-occurrence statistical information, International Journal on Artificial Intelligence Tools, 13(01), pp. 157-169.

Ortuno, M. et al. 2002. Keyword detection in natural languages and DNA, EPL (Europhysics Letters), 57(5), p. 759.

Rényi, A .1961. On measures of entropy and information, in Proceedings of the Fourth Berkeley Symposium on Mathematical Statistics and Probability, Volume 1: Contributions to the Theory of Statistics.

Rossi, R. G., Marcacini, R. M. and Rezende, S. O. 2014. Analysis of domain independent statistical keyword extraction methods for incremental clustering, Learning and Nonlinear Models, 12(1), pp. 17-37.

Shannon, C. E. 1948. A mathematical theory of communication, Bell system technical journal, 27(3), pp. 379-423.

Siddiqi, S. and Sharan, A. 2015. Keyword and keyphrase extraction techniques: a literature review, International Journal of Computer Applications, 109(2).

Tsallis, C. 1988. Possible generalization of Boltzmann-Gibbs statistics, Journal of statistical physics, 52(1-2), pp. $479-487$. 